\title{
Unobtrusive Sleep Stage Identification Using a Pressure-Sensitive Bed Sheet
}

\author{
Lauren Samy, Ming-Chun Huang, Jason J. Liu, Senior Member, IEEE, Wenyao Xu, \\ and Majid Sarrafzadeh, Fellow, IEEE
}

\begin{abstract}
Sleep constitutes a big portion of our lives and is a major part of health and well-being. Monitoring the quality of sleep can aid in the medical diagnosis of a variety of sleep and psychiatric disorders and can serve as an indication of several chronic diseases. Sleep stage analysis plays a pivotal role in the evaluation of the quality of sleep and is a proven biometric in diagnosing cardiovascular disease, diabetes, and obesity [32]. We describe an unobtrusive framework for sleep stage identification based on a high-resolution pressure-sensitive e-textile bed sheet. We extract a set of sleep-related biophysical and geometric features from the bed sheet and use a two-phase classification procedure for Wake - Non Rapid Eye Movement Rapid Eye Movement stage identification. A total of seven allnight polysomnography recordings from healthy subjects were used to validate the proposed bed sheet system and the ability to extract sleep stage information from it. When compared with the gold standard, the described system achieved $70.3 \%$ precision and $71.1 \%$ recall on average. These results suggest that unobtrusive sleep macrostructure analysis could be a viable option in clinical and home settings in the near future. Compared with existing techniques for sleep stage identification, the described system is unobtrusive, fits seamlessly into the user's familiar sleep environment, and has additional advantages of comfort, low cost, and simplicity.
\end{abstract}

Index Terms-Sleep staging, sleep quality, polysomnography, respiratory rate, bed sheet, pressure sensor array, unobtrusive, e-textile.

\section{INTRODUCTION}

$\mathbf{T}$ HOUGH once considered an inactive state in which the body and brain simply shut down, sleep is now recognized as a phase of high mental activity that fulfills necessary biological functions. The vital tasks carried out during sleep are essential for our physical, mental and emotional health. Studies have shown that the quality of sleep, rather than its quantity, is highly correlated with health and well-being [26]. Sleep quality analysis is, therefore, gaining importance and being used in the diagnosis of diverse health problems.

Sleep medicine divides sleep into two broad classes: Nonrapid eye movement (NREM) and rapid eye movement (REM)

Manuscript received July 1, 2013; revised August 29, 2013 and October 13, 2013; accepted November 11, 2013. Date of publication December 5, 2013; date of current version May 22, 2014. The associate editor coordinating the review of this paper and approving it for publication was Dr. Stefan Bosse.

L. Samy is with the Department of Computer Science, University of California, Los Angeles, CA 90095 USA (e-mail: 1samy@cs.ucla.edu).

M.-C. Huang, J. Liu, W. Xu, and M. Sarrafzadeh are with Wireless Health Institute, University of California, Los Angeles, CA 90095 USA (e-mail: mingchuh@cs.ucla.edu; jasonliu@cs.ucla.edu; wenyao@cs.ucla.edu; majid@cs.ucla.edu).

Color versions of one or more of the figures in this paper are available online at http://ieeexplore.ieee.org.

Digital Object Identifier 10.1109/JSEN.2013.2293917 sleep. The American Academy of Sleep Medicine (AASM) further divides NREM sleep into three distinct stages: N1, $\mathrm{N} 2$, and N3 [30]. In healthy adults, a sleep cycle, which lasts between 90 and 100 minutes, begins with 3 stages of NREM sleep followed by REM sleep. The first stage (N1) is the lightest and shortest stage of sleep (1-7 minutes) and marks the transition from wakefulness (W) to sleep. N1 is followed by $\mathrm{N} 2$, which lasts anywhere from 10 to 25 minutes. This stage is where the body reaches a state of complete relaxation in preparation for the deeper sleep to come. After $\mathrm{N} 2$, a healthy adult enters $\mathrm{N} 3$, the last stage of NREM which is also referred to as deep sleep. N3 lasts 20 to 40 minutes and is the stage where the body does most of its repair and regeneration work. Following the N3 stage of sleep, a healthy adult ascends to lighter NREM sleep stages, typically N2, for 5 to 10 minutes before entering the REM sleep episode. REM sleep is characterized by high brain activity and is where memory consolidation occurs. REM sleep comprises about 20 to $25 \%$ of total sleep in typical healthy adults [4], [18]. In healthy subjects, this pattern continues to repeat in a cyclical fashion throughout the night. Since each stage fulfills a vital biological function, sleep stage analysis is crucial to the evaluation of the quality of sleep and is a proven biometric in diagnosing cardiovascular disease, diabetes, and obesity [32].

In sleep medicine, polysomnography (PSG) is a sleep test that is conducted in a specialized sleep laboratory to assess sleep stages and other characteristics of sleep quality. Measurements taken include EEG (brain waves), EOG (eye movement), EMG (muscle activity), and ECG (heart rhythm) to mention a few. The standard practice is to divide the sleep time into 30-second epochs, and based on the recorded signals, each epoch can be scored by sleep technicians as W, N1, N2, N3 or REM. Since the pattern of the Wake-NREM-REM stages is highly modified for subjects with sleep disorders and other diseases, sleep stage analysis can provide valuable information about sleep quality. The very nature of polysomnography, however, can interfere with the results and accuracy of the measured signals. The unfamiliar environment of the sleep laboratory as well as the equipment and sensors attached to the subject's body can disturb the subject's quality of sleep - a phenomenon referred to as the first-night effect [31]. Thus, a system able to non-invasively evaluate sleep quality can contribute to more convenient and accurate sleep screening results and is highly desirable.

Our contributions in this work can be summarized as follows. To the best of our knowledge, our work is the first 
to perform sleep stage analysis using a completely contactfree unobtrusive system. In addition, our sleep stage analysis results are validated against over 50 hours of gold standard polysomnography data - the current state-of-the-art in sleep analysis. Finally, our results are in the range of interrater agreements reported in the literature (70\% and $72 \%$ [11]) which is very promising.

The remainder of the paper is organized as follows. Section 2 briefly discusses previous research in sleep stage detection. Section 3 describes the components of our system which incorporates a pressure-sensitive bed sheet. Section 4 describes the algorithm used for sleep stage identification, including biophysical and geometric feature extraction from pressure images and a two-phase procedure used for classification. In section 5, the experimental setup and results are demonstrated, followed by our planned future work in section 6. Section 7 concludes the paper.

\section{RELATED WORK}

While there are many academic and commercial sleep monitoring tools available, there is a dearth of low-cost, unobtrusive solutions. This section surveys the major categories of solutions and describes their characteristics and limitations.

Existing sleep monitoring tools can be classified into three categories. The first category of tools extracts sleep stages from directly-measured physiological signals. Because many body functions like breathing, heart rate and movement change during sleep, tracking these changes throughout the night can provide a good indication of the sleep stage that a patient is in. Many tools distinguish themselves from full PSG by using only a small subset of the PSG sensors. [28] shows that ECG and respiratory effort alone can be sufficient to distinguish between the WAKE, NREM and REM stages with moderate accuracy. [13] uses features extracted from the EEG signal in addition to heart rate variability to detect the different sleep stages. [14], on the other hand, uses only the EEG signal to study brain activity and determine sleep stages based on it. Another example is Zeo [8], a commercial sleep monitoring product that detects sleep stages based on brain activity. A headband, to be worn during sleep, analyzes brain signals and identifies sleep stages based on the signals' frequencies. Although these systems use fewer sensors than a full PSG, they still require equipment to be attached to the patient's body and are often expensive.

The second category of tools tries to infer sleep stages from body movement. Actigraphy is a commonly used technique for sleep monitoring; it uses an accelerometer embedded in a watch-like device to monitor activity and identify sleep stages [12], [15], [29]. Sleeptracker [6], Actiwatch [5], and UP [1] are a few examples of the many commercial products available in this category. Though less invasive than the products in the first category, these products still require a device to be attached to the patient's wrist. To overcome this limitation, [16] presents a sleep monitoring system based on RFID technology. WISP tags are added along the edge of the bed mattress and accelerometer data is collected from these tags by an RFID reader. This system does not require any attachment to the patient's body but might represent a problem if it were to be deployed in a clinical setting since the RFID frequency can interfere with other medical devices [34].

The third category of products uses audio and video signals to identify sleep stages. [25] uses audio and video sensors to infer sleep-wake stages. These systems are expensive and raise obvious privacy concerns. In [9], the authors attempted to solve the privacy problem by using an infrared camera. They analyze the temperature maps acquired from the camera to detect the patient's body and to extract body movement information. Infrared cameras are a controversial technology, however, as the high-resolution thermal images make it very simple to observe exactly what the patient is doing in bed and can be considered a breach of privacy.

Our proposed system opens the way for a fourth category of sleep monitoring that is completely unobtrusive, comfortable, cheap, and avoids privacy violations. The system architecture of this new unobtrusive system is described in the next section.

\section{System ARCHITECTURE}

In this section, we describe the design of our bed sheet system, followed by a detailed description of the PSG system that was used for validation.

\section{A. Bed Sheet System}

E-textiles are fabrics that have electronics and interconnections woven into them. E-textiles are built by sandwiching a sheet of very thin piezoresistive fabric between two sheets of traditional textile fabric [33]. The middle e-textile layer has a couple of useful characteristics: 1) the electrical resistance of the piezoelectric fabric decreases with increasing pressure force, 2) the fabric's thickness is on the order of microns, comparable to the diameter of a human hair. These two characteristics enable us to build a highly flexible, comfortable and low-cost pressure-sensing system that perfectly fits our sleep monitoring application.

The proposed system consists of an e-textile bed sheet that records the pressure distribution of the body. The prototype bed sheet is $1.25 \mathrm{~m}$ wide $\times 2.5 \mathrm{~m}$ long and can fit easily on any standard-size bed. The thickness of the bed sheet is $1.5 \mathrm{~mm}$ which makes it flexible and suitable for noninvasive applications. The bed sheet is a matrix of 8192 pressure sensors generated by the intersections of 64 columns and 128 rows of conductive buses.

To build the sensors, we use a three-stacked-layer structure as shown in Fig. 1. The top layer is a conductive bus that is connected to a voltage supply via an analog multiplexer $\left(M_{1}\right)$. The middle layer consists of the pressure-sensitive e-textile piezoresistive fabric. Lastly, the bottom layer is a conductive bus that is orthogonal to the top bus. This bottom layer connects to an analog-to-digital converter (ADC) via a second analog multiplexer $\left(M_{2}\right)$ such that each input to the ADC is grounded via an offset resistor $\left(R_{0}\right)$. The intersection areas between the orthogonal buses form the individual sensors. A microcontroller is used to set the addresses of both $M_{1}$ and $M_{2}$ to uniquely select a pressure sensor. For example, when $M_{1}$ connects bus i on the top layer to a voltage supply 


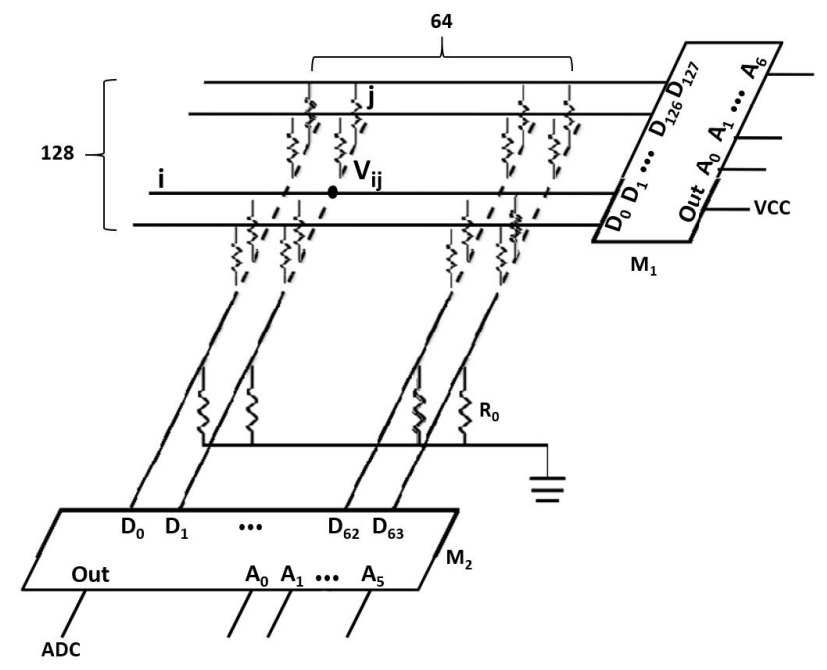

Fig. 1. The circuit used to scan the pressure distribution of the bed sheet sensor array.

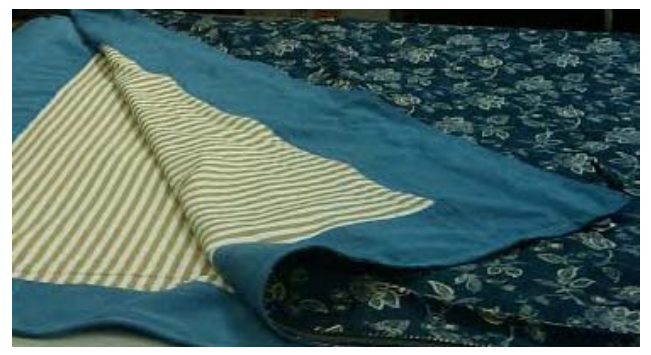

Fig. 2. Cross section of the three-stacked-layer structure of the e -textile bed sheet. The e-textile piezoresistive fabric is sandwiched between two orthogonal conductive bus layers.

and $M_{2}$ connects bus $\mathrm{j}$ to the ADC, the sensor located at row $\mathrm{i}$, column $\mathrm{j}$, which is denoted as $V_{i j}$ in Fig. 1, will be read. The ADC converts the voltage of the selected sensor to an 8-bit integer with a value of 0 representing no pressure (highest e-textile resistance) and a value of 255 representing maximum pressure (lowest e-textile resistance). The sensor values are then sent to an Android tablet over USB for storage and further analysis. Fig. 2 shows a cross section view of the bed sheet. The e-textile piezoresistive fabric is sandwiched between two orthogonal conductive bus layers, as described above.

The advantage of this design is that the top and bottom layers can be made out of traditional fabric coated with parallel conductive buses with the e-textile piezoresistive fabric embedded in between. Another advantage of this design is that the $\mathrm{MxN}$ sensor structure only requires $2(\mathrm{M}+\mathrm{N}) \mathrm{I} / \mathrm{O}$ pins. Also, the matrix structure of the bed sheet enables random access to an arbitrary sensor in the system.

Fig. 3(a) shows an example of a user lying on the bed sheet and Fig. 3(b) shows the corresponding pressure map. Compared to other systems that use bed mattresses, sheets or pads for health applications [21], [23], the described system has the flexibility of e-textiles as well as a dense highresolution pressure map that enables high-quality medical diagnosis.

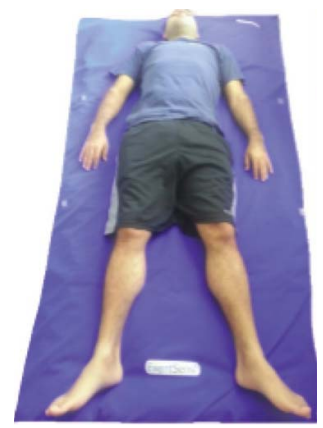

(a)

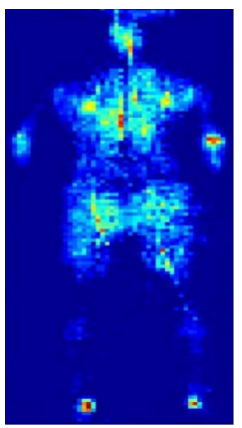

(b)
Fig. 3. (a) A subject sleeping on the pressure-sensitive bed sheet in the supine position. (b) The corresponding pressure map.

\section{B. PSG System}

The reference gold standard used for assessing the accuracy of our sleep identification algorithm is the SOMNOscreen plus PSG system manufactured by SOMNOmedics [7]. The PSG system has a comprehensive set of sensors that allows it to monitor many body functions and accurately identify sleep stages. Fig. 4 shows all the PSG sensors and their respective locations on the subject's body. Fig. 4(a) shows 8 EEG electrodes that are attached to the subject's head to measure brain activity. The electrodes measure the frontal (red), central (blue), medial (yellow) and occipital (green) EEG signals which have different characteristics and frequencies during the various stages of sleep. Fig. 4(b) shows face and neck sensors. One electrode is attached to the middle of the forehead for grounding. To measure eye movement, two EOG electrodes are attached near the eyes; one is attached above the right eye and the other is attached below the left eye. Three EMG electrodes are attached to the subject's chin to detect chin muscle movement which can be indicative of teeth grinding (bruxism), sleep apnea and other sleep disorders [3]. The two EMG electrodes to the left and right of the chin midline are the negative and positive leads, respectively, while the one in the middle of the chin is the reference EMG lead. A thermistor is placed directly below the nostrils. The thermistor measures the nasal and oral flow of the patient. In addition, a nasal cannula is placed on top of the thermistor and directly inside the nostrils. This sensor detects the fluctuations in pressure caused by inhalation and exhalation. A microphone is placed on the neck, lateral to the larynx, to detect snoring. Fig. 4(c) shows the sensors that are attached to the upper body. Two ECG electrodes are used to measure the rate and regularity of heartbeats. One electrode is attached under the right clavicle (collarbone) and the other one is attached under the rib cage on the left side of the body. Two effort belts are attached around the thorax and abdomen to measure the expansion of the chest and abdomen during breathing. Finally, Fig. 4(d) shows the lower body sensors. A pulse oximeter is attached to the ring finger to measure blood oxygen saturation, heart rate, and changes in lung volume. A pair of EMG active electrodes are attached to the legs centered between the knee and the ankle to record leg movement. All the sensors are plugged in to the central PSG system, which in turn is 


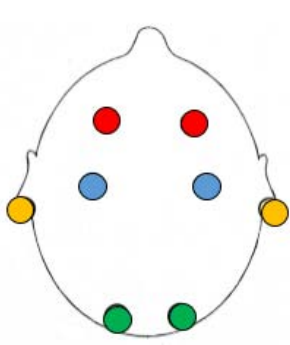

(a)

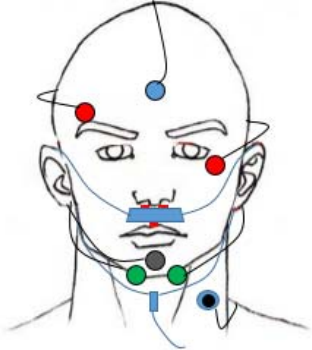

(b)

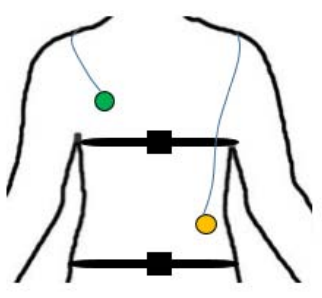

(c)

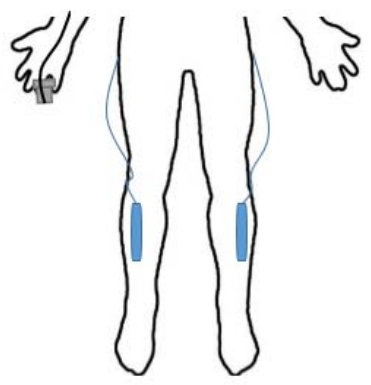

(d)

Fig. 4. PSG sensors and their placement on the head(a), face(b), upper body(c), and lower body(d).

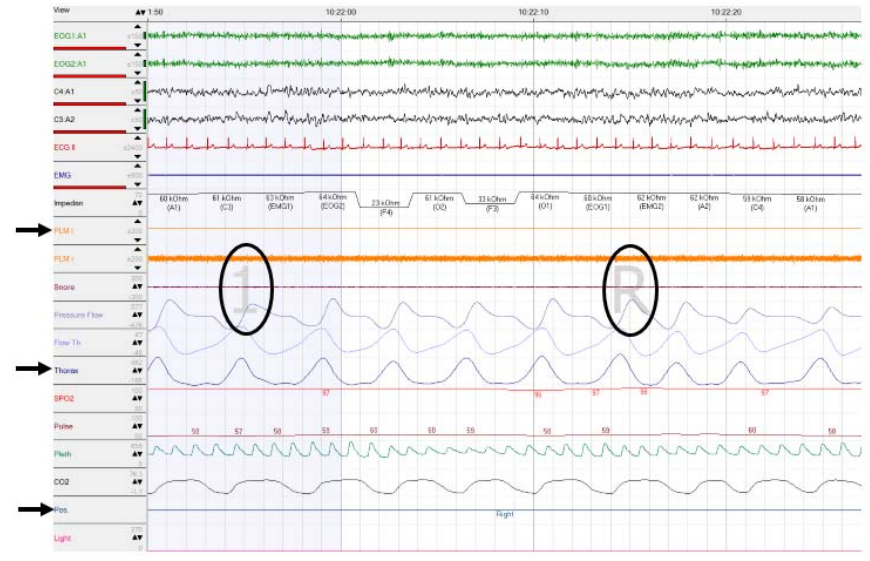

Fig. 5. A sample polysomnogram that shows a subject transitioning from sleep stage N1 to REM as indicated by the circles. The polysomnogram shows 19 of the PSG signals and the three arrows are pointing to the three channels that are used as ground truth for leg movement (PLM), respiration (Thorax) and whole-body movement (Posture).

connected to a Bluetooth transmitter. The transmitter sends the data wirelessly and in real time to a receiver connected to a laptop that runs the PSG analysis software. Fig. 5 shows a sample polysomnographic record as provided by the analysis software.

While the PSG system accurately captures all the necessary body functions, it is clear to see how the attached sensors can disturb the patient's sleep and restrict movement and thereby affect the quality of sleep. In the next section, we describe how to identify sleep stages using our unobtrusive bed sheet system.

\section{Analysis Methods}

In our study, three statistical classifiers were used in a twophase procedure to classify a sleep epoch to the NREM, Wake or REM sleep stage. The parameters for each classifier were obtained by learning from our training dataset. In each phase of classification, each target/non-target sample was labeled with the binary values 1 or 0 , respectively; each classifier was used to assign a testing data point - in our case a sleep epoch - to one of these two labels which map to two different sleep stages. The overall accuracy is determined by the percentage of the testing data that was assigned to the correct sleep stage. The three classifiers used in this work are based on different statistical principles and are described below.

The binary Support Vector Machine (SVM) classifier is a non-probabilistic linear classifier. It constructs an optimized hyper-plane in the feature space such that the separation between two different types of samples is maximized. The hyper-plane is obtained by learning from the training samples. Each training sample contains a binary label to indicate the group it belongs to. Once the hyper-plane is calculated, the testing data can be projected onto the feature space and classified by the hyper-plane into one of the two categories.

The K-Nearest-Neighbor classifier uses the notion of distance between data points in the feature space as the basis for classification. It assigns a testing data point to the class which the majority of the $\mathrm{k}$ nearest neighbors are from. Similar to SVM training, the labels of the nearest neighbors are obtained from the training data. In this work, Euclidean distance is used as the distance metric and the majority rule is applied to the $\mathrm{k}$ candidates in order to determine the group that the sample belongs to.

Naive Bayes is a probabilistic classifier based on the Bayesian theorem. It is particularly suitable when the dimensionality of the inputs is high. It assumes that features contribute independently to the probability of a given sample belonging to a certain class. Bayes' Theorem finds the probability of an event given the probability of another event that has already occurred.

\section{Sleep Stage Identification}

In this section, we describe the features used for classification, how they are extracted from the bed sheet pressure images and why they are suitable for sleep stage identification. The pressure images from the entire night are divided into 30-second epochs, as is the convention in sleep medicine, and the features are extracted for each epoch. Following feature extraction, we describe the two-phase procedure used for identifying the Wake, NREM and REM stages.

\section{A. Biophysical Feature Extraction}

As discussed in Section 2, several physiological signals change during normal sleep and vary with each sleep stage. Three important signals for sleep stage identification are 


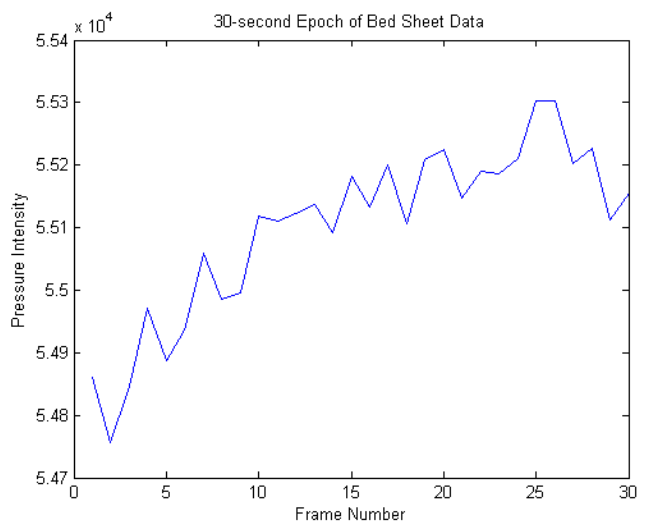

Fig. 6. Pressure signal corresponding to respiration over a 30-second epoch.

respiratory effort, leg movement and body movement. The following subsections describe each one in more detail and explain how they were extracted from the pressure images.

1) Respiration Rate Variability: Respiration is a physiological signal that undergoes significant changes during sleep. During the NREM sleep stage, the breathing pattern is regular, both in amplitude and frequency, while in REM the signal becomes irregular, much more rapid, and sudden changes in both amplitude and frequency can be observed [20].

To capture this behavior of the respiratory signal, we first extract the respiration signal from the bed sheet. When the user lays on the bed sheet and starts breathing, the movement of the diaphragm resulting from inhalation and exhalation causes the observed pressure values to decrease and increase, respectively. These alternating movements of the chest area are significant enough to cause a substantial change in the overall pressure intensity of a pressure image. The pressure intensity of an image can be calculated by summing up the individual pressure values from each one of the 8192 pressure sensors (pixels) in that image. Fig. 6 shows a plot of the pressure intensity signal for a user in the supine position over the period of the first 30-second epoch. Since the sampling rate is $1 \mathrm{~Hz}$, this also corresponds to 30 frames. When the user laying on the bed sheet inhales, his/her chest will rise up towards the ceiling and the pressure applied to the bed sheet underneath will decrease. Therefore, each local minimum in the figure represents a drop in pressure or, alternatively, an inhalation. The figure also shows the drifting phenomenon. Drift is an inherent property of pressure sensors that often causes a signal to grow logarithmically over time until the system reaches operating temperature [24]. Such drift is a significant source of noise and needs to be compensated for to improve the quality of the signal. In our previous work, we showed how first-order derivation can be exploited to compensate for the drifting phenomenon [17]. Fig. 7 shows the resulting signal after first-order derivation is applied. To eliminate short-term fluctuations, we apply a low-pass filter to the signal to get the final signal shown in Fig. 8.

After drift compensation and low-pass filtering are applied, the resulting signal is used to evaluate the accuracy of our bed sheet system in extracting respiration. To evaluate the

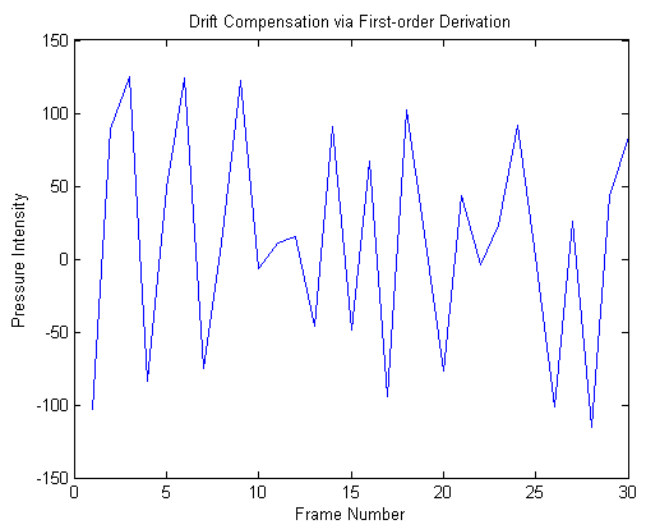

Fig. 7. Pressure signal corresponding to respiration over a 30-second epoch after drift compensation. As can be seen, the logarithmic growth of the respiration signal is now eliminated.

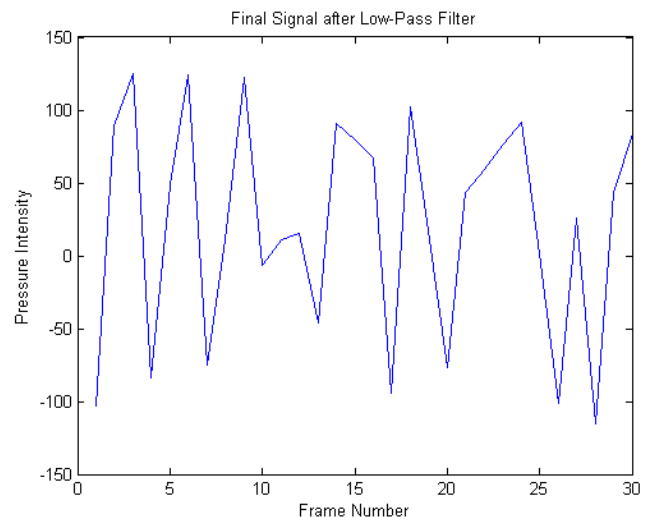

Fig. 8. The figure shows the respiration signal after low-pass filtering is applied. As can be seen, low-pass filtering eliminated short fluctuations in the signal and produced a smoother respiration signal.

respiration signal obtained from the bed sheet, we use the thorax effort signal obtained from the PSG system as ground truth. The thorax signal is generated by measuring the amount of pressure applied to the effort belt that the user wears around the chest. The thorax effort signal is shown in Fig. 9. It is noteworthy to mention that, in contrast to the pressure signal obtained from the bed sheet, this signal indicates an inhalation event at each local maximum. The signals are inverted because when a user inhales and his/her chest rises, the pressure exerted on the bed sheet decreases while the pressure exerted on the effort belt increases. Another difference between the two signals under comparison is the sampling rate. While the bed sheet pressure image is sampled at 1 pressure image per second, the thorax effort signal has a sampling rate of $32 \mathrm{~Hz}$. While Fig. 6 shows 30 samples on the x-axis, Fig. 9 shows 960 samples for the same period of time. As shown in the figure, the thorax effort signal indicates a total of 8 inhalations and 8 exhalations (local maxima and minima, respectively), for a total of 8 breaths. During the same time period, the pressure signal obtained from the bed sheet indicates the same number of inhalations and exhalations. Both systems would therefore result in a respiration rate of 8 breaths per 30 seconds, or 16 breaths per minute. 


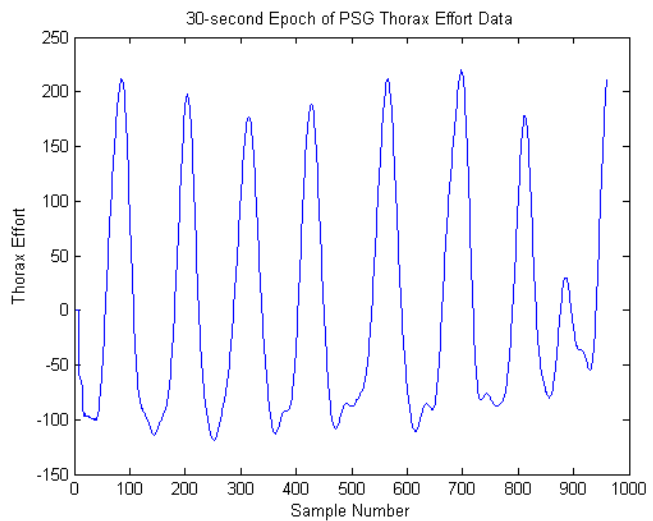

Fig. 9. The figure shows the thorax effort signal obtained from the PSG system. Each local maximum indicates an expansion of the chest which corresponds to an inhalation event.

After extracting a filtered drift-free respiration signal, we compute the mean amplitude and the mean frequency of that signal in each 30-second epoch. Then, for each portion of the signal in the 30-second epoch, we compute its variability from the mean in terms of both amplitude and frequency. This is done as follows. Starting from the first sample in the signal, we compute its standard deviation from the mean amplitude and the mean frequency of that epoch. We incrementally grow the size of the signal by adding one sample at a time and computing the amplitude standard deviation and the frequency standard deviation until all 30 samples of an epoch are covered. We then move to the next epoch and repeat the process. That way, we get a measure of the dispersion of the respiration signal from the mean amplitude and the mean frequency of an epoch at each pressure frame.

The variability of the respiration signal is a very good feature to distinguish between the NREM stage where the signal is regular and shows very little variability, and the Wake-REM stages where the amplitude and frequency of the respiration signal vary significantly and rapidly.

2) Respiration Rate: Respiration rate, as measured by the number of breaths observed per minute, is another biophysical signal that changes during sleep. Respiration rate is considerably faster during the REM and Wake sleep stages than in the NREM stage. So, an additional respiratory feature used for sleep stage identification is the respiration rate observed during a 30 -second epoch. The breathing rate is extracted from the respiratory signal by counting the number of breaths as described above and multiplying by two to get the number of breaths per minute.

3) Leg Movement: Leg movement is also an important indicator of sleep stage. During the first stage of NREM, sleepers can experience sudden jerks of their legs. These jerks are common while falling asleep but, if excessive, can also be a symptom of sleep disorders like Periodic Limb Movements (PLM) and Restless Legs Syndrome (RLS) [10]. In either case, these jerks are associated with the NREM sleep stage and can be used as a feature to distinguish the NREM sleep stage from the other stages.

To extract leg movement from the bed sheet, we assume that a subject's legs while lying on the bed sheet will occupy the lower half of the sheet. To extract leg movement, we sum all the pixels in the lower half of the pressure image and mark a leg movement when a significant drop or increase in pressure is detected. This is a crude approximation of leg movement and can be improved in the future by localizing the patient's legs in the pressure images. The ground truth for leg movement events is obtained from the PLM channel of the PSG recording, as shown in Fig. 5. This signal measures leg muscle movement over time. The signal is obtained by sampling the leg EMG electrode at $256 \mathrm{~Hz}$. When compared to the PLM signal obtained from the PSG system, our approximation accurately detects $80.7 \%$ of all leg movements.

4) Body Movement: In addition to leg movement, wholebody movement can also occur during sleep. The movements are associated with the Wake stage and light stages of NREM as a result of changes in sleep posture that occur every 5-10 minutes. REM sleep, on the other hand, is characterized by muscle immobility and body paralysis to prevent sleepers from acting out their dreams and hurting themselves [27]. Therefore, the lack of movement can be a good indication of the REM sleep stage.

During movements, certain body parts like arms, hands, elbows or knees are lifted off the bed sheet. This abrupt release in pressure results in a significant drop in pressure intensity. This sudden and significant change in pressure can be detected by a simple thresholding technique that keeps track of the difference between local maxima and minima over a sliding window and reporting a movement if the difference drops significantly. Our algorithm reports a movement if there is a change of more than an order of magnitude in this peak-to-peak amplitude. The detected movements are validated against the posture information provided by the PSG system. Our thresholding algorithm correctly detects $96.5 \%$ of the whole-body movement events.

5) Posture and Body Orientation Features: In addition to the biophysical features described above, we also extracted some geometric features from the pressure images. The geometric features are motivated by the fact that the orientation of the body during sleep as well as sleep posture can affect sleep stages. This is mainly because we are likely to go into NREM and the deeper stages of sleep if our bodies are situated in a comfortable position, especially when the body is in a "mid-line" position, where both the head and neck are kept straight.

Furthermore, according to the British Snoring and Sleep Apnoea Association [2], patients who sleep in the side positions often demonstrate a decrease in the amount of NREM and an increase in the amount of REM sleep.

We used 32 geometric features including body symmetry, balance, hip location and shoulder location. These features summarize posture and body orientation and are explained in more detail in [22].

\section{B. Sleep Stage Identification Using Two-Phase Classification}

To perform sleep stage identification, the pressure images from a full-night sleep are divided into groups of 30 frames. 


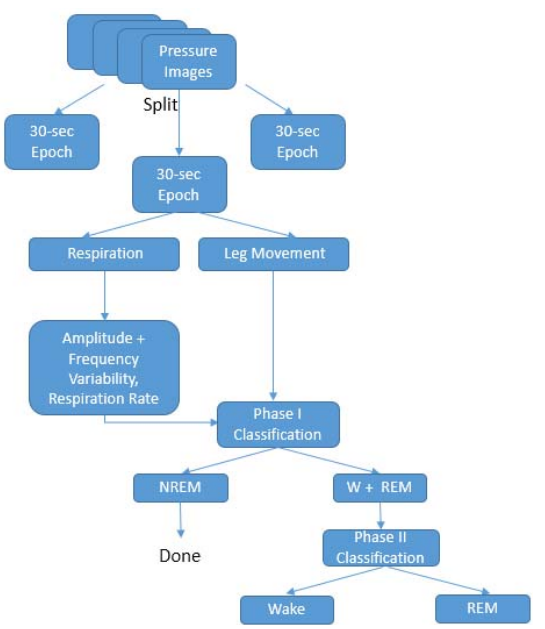

Fig. 10. This figure shows the steps of the sleep stage identification process. The pressure images of a whole night are split into 30-second epochs from which biophysical signals are extracted. Phase I classification groups epochs into NREM and Wake+REM. In Phase II, the Wake+REM epochs are then classified separately based on the extracted movement information.

Since the sampling rate for the bed sheet is $1 \mathrm{~Hz}, 30$ frames corresponds to 30 seconds of sleep. It is common in polysomnography analysis to split the night into 30 -second epochs, so the same practice was followed in this work. For each 30-second epoch, the amplitude and frequency variability of the respiratory signal, the respiration rate, the leg movement events as well as the geometric features described in Section 4.1 are extracted. Since those features mostly distinguish the NREM stage from the rest of the stages, these features are used for phase I classification into NREM and Wake + REM. Phase I, therefore, acts as a filter for the NREM sleep stage. In phase 2, the algorithm tries to distinguish between the Wake and REM stages. Since body movement cannot occur in the REM stage, as mentioned before, it can be used as a feature to distinguish between Wake and REM. For all the epochs that were classified as Wake + REM in phase I of the algorithm, body movement events are extracted and used to determine if a given epoch should finally be classified as Wake or REM. The decision tree for the described process is shown in Fig. 10.

\section{EXPERIMENTS AND RESULTS}

In this section, we describe our experimental setup and show the results of our sleep stage identification process.

\section{A. Experimental Setup}

Seven subjects participated in the sleep study. Three of the subjects were female, four were male. Their ages ranged from 21 to 60 years and their weights from 93 to 190 pounds. Each subject underwent a full-night PSG study and had all the sensors described in Section 3.2 attached to him/her. At the same time, each subject slept on the e-textile bed sheet and his/her pressure images were continuously recorded overnight. The 50+ hours of PSG recordings from the seven subjects were scored by SOMNOmedic's Domino software. The thorax effort signal, leg EMG signal, sleep posture and sleep stage

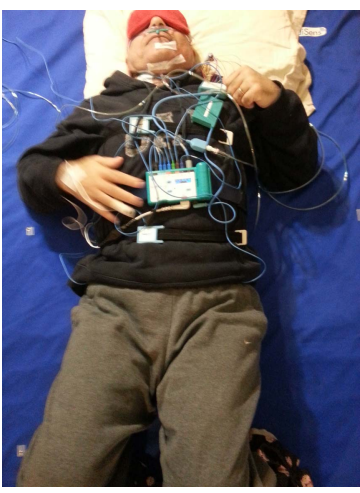

Fig. 11. A male patient lying on the bed sheet with all PSG sensors attached to his body shortly before the lights were turned off.

information provided by the software served as ground truth for all extracted features. The experiments for all 7 subjects were conducted under similar conditions. To ensure the setting was comfortable for sleeping, the light was turned off, the air in the room was in circulation and nearby noise sources were eliminated. The room temperature was set to 68 degrees Fahrenheit which is a comfortable temperature for sleeping. Fig. 11 shows a subject wearing the PSG sensors and laying down on the bed sheet shortly before the lights were turned off.

Once the patient got in bed and before starting the overnight recording, the so-called bio-calibrations were performed. Biocalibrations are short tests whose purpose is to discover any poor PSG signals or incorrect hookups prior to the start of the study. The subjects were asked to blink 5 times in order to test the EOG signal. They were then asked to point their toes towards their nose. This bio-cal tests the EMG electrodes placed on the legs. Clenching the teeth verifies the accuracy of the chin EMG signal, and breathing through the nose and mouth verifies the flow, pressure and effort signals. To test the microphone, the patient was asked to make a snoring sound and to verify the posture detection provided by the PSG system, the subject was asked to change the sleeping position. In the morning and before disconnecting the sensors, the same bio-calibrations are repeated to ensure that all the sensors remained in place throughout the night. One out of the 7 allnight PSG recordings had a problem with the morning biocalibrations; the thorax effort belt loosened during the night, resulting in a poor respiration signal. That recording had to be repeated the following night.

The pressure images obtained from the bed sheet were labelled using the sleep stage information provided by the PSG software. Testing was done using Leave One Out Cross Validation (LOOCV). One subject's pressure images are left out for testing and the other subjects' images are used for training.

\section{B. Results}

Figure 12 shows the performance results of our sleep stage identification process based on signals derived from the bed sheet. The results of three different classifiers after phase I and phase II, as well as the overall results, are shown. 


\begin{tabular}{|c|c|c|c|c|c|}
\hline Classifier & Precision & Recall & Phase I Accuracy & Phase II Accuracy & Total Accuracy \\
\hline K-Nearest Neighbor & $55.9 \%$ & $56.9 \%$ & $68.75 \%$ & $65.67 \%$ & $67.12 \%$ \\
\hline Support Vector Machines & $67.7 \%$ & $68.6 \%$ & $73.10 \%$ & $68.50 \%$ & $70.33 \%$ \\
\hline Naive Bayes & $70.3 \%$ & $71.1 \%$ & $75.23 \%$ & $69.20 \%$ & $72.20 \%$ \\
\hline
\end{tabular}

Fig. 12. Comparison of different classifiers in terms of precision, recall, phase I performance, phase II performance, and overall accuracy.

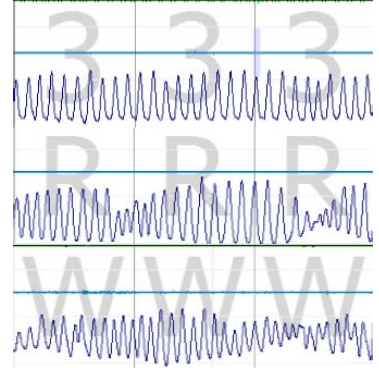

Fig. 13. This figure shows the respiration signal during three different epochs: one epoch during the N3 stage of NREM (top), another epoch during REM (middle) and yet another during Wake (bottom).

It is noteworthy to mention that the Naive Bayes classifier outperforms both SVM and KNN in terms of precision, recall and accuracy despite the oversimplified assumptions it makes about the underlying probability model of the data. This is likely the case because in each of the two phases of classification, the correct class is more probable than the other class. In phase I, for example, where we try to separate the NREM stage from the other stages, classifying more samples as NREM than REM+Wake is likely to produce good results since the NREM stage constitutes $75 \%-80 \%$ of the night and is therefore more probable. The same argument can be made for phase II.

Another point to observe is that the performance of phase II is significantly worse than the performance of phase I. This performance degradation is expected since the physical changes that occur during REM sleep are very similar to the ones that occur during the Wake stage, making the task of separating REM and Wake epochs in phase II a challenging task. Because of the similarity between the REM and Wake stages, the REM sleep stage is sometimes referred to as paradoxical sleep. Even though it is one of the stages of sleep, it is characterized by a brain wave pattern and physical signals that are similar to that of wakefulness. Fig. 13 visually shows the similarity between REM and Wake, as well as the dissimilarity between those two stages and the NREM stage. The figure shows the respiratory signal acquired by the PSG system during three epochs of the N3 stage of NREM (top), REM (middle) and Wake (bottom) of a single patient. As can be seen, the respiratory signal in the NREM stage is regular with very little variation in amplitude and frequency. Both the REM and Wake stages, on the other hand, show the same irregularity with clear variations in the amplitude and frequency of the respiratory signal.

Fig. 14 shows the hypnogram of one of the seven subjects. A hypnogram is a graph commonly used in polysomnography that represents the stages of sleep as a function of time, specifically as a function of epochs. In a clinical setting, a
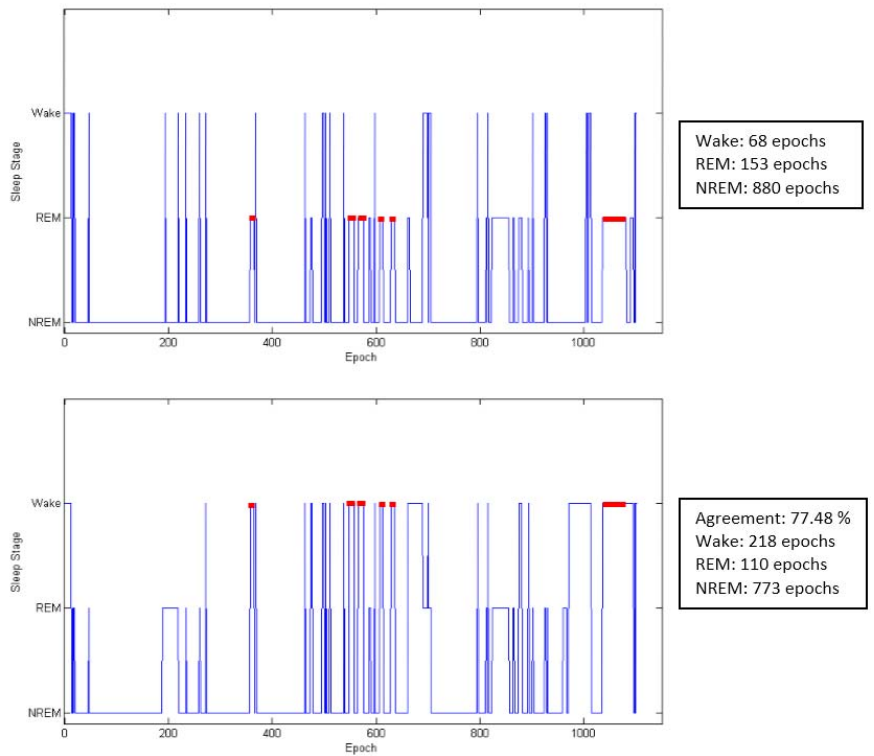

Fig. 14. The top hypnogram shows sleep stages over time as provided by the PSG system. (Wake: 68 epochs, REM: 153 epochs, and NREM:880 epochs). The bottom hypnogram shows the hypnogram as obtained by the two-phase classification procedure. (Wake: 218 epochs, REM: 110 epochs, and NREM: 773 epochs, Agreement: $77.48 \%$ ).

polysomnography record is usually scored by more than one sleep technician and the hypnogram provides a visual way to show the agreement between the scoring of the different technicians. Here, we use a hypnogram to visually show the agreement between the sleep stages obtained from the PSG system (top) and the ones obtained from the bed sheet after feature extraction and classification (bottom). The classifier used in the creation of this hypnogram is SVM. The hypnogram from the bed sheet shows $77.48 \%$ agreement with the gold-standard hypnogram. It can be observed from the figure that a significant majority of the NREM epochs are classified correctly as indicated by the matching NREM portions of the hypnograms. It can also be observed that the REM and Wake stages are often misclassified. The red bolded lines in the bottom hypnogram show the epochs that were incorrectly classified as Wake as a result of our classification algorithm. The red bolded lines in the top hypnogram show that these misclassified epochs were scored as REM epochs by the PSG system.

Finally, Fig. 15 shows two confusion matrices for the same patient as in the hypnograms. The top confusion matrix shows the precision and recall values for the two-phase classification algorithm described in section 4.2. Again, we can observe that phase II of the classification performs poorly compared to phase I due to the similarity of the REM and Wake stages. If no body movements occur during the Wake stages then separating 


\begin{tabular}{|c|c|c|c|c|}
\hline & NREM & REM & Wake & Recall \\
\hline NREM & $\mathbf{7 7 3}$ & 38 & 69 & $87.8 \%$ \\
\hline REM & 0 & $\mathbf{4 2}$ & 111 & $27.5 \%$ \\
\hline Wake & 0 & 30 & $\mathbf{3 8}$ & $55.9 \%$ \\
\hline Precision & $100 \%$ & $38.2 \%$ & $17.4 \%$ & $\mathbf{7 7 . 5} \%$ \\
\hline
\end{tabular}

\begin{tabular}{|c|c|c|c|c|}
\hline & NREM & REM & Wake & Recall \\
\hline NREM & $\mathbf{7 2 9}$ & 76 & 113 & $79.4 \%$ \\
\hline REM & 2 & 12 & 139 & $7.8 \%$ \\
\hline Wake & 27 & 25 & 16 & $23.5 \%$ \\
\hline Precision & $96.2 \%$ & $10.6 \%$ & $6.0 \%$ & $\mathbf{6 8 . 8} \%$ \\
\hline
\end{tabular}

Fig. 15. The top confusion matrix shows the results when the SVM classifier is used in two-phase classification. The bottom confusion matrix shows the results when the SVM classifier is used in one-phase classification on the same data.

REM and Wake becomes even more difficult. The bottom confusion matrix corresponds to a one-phase classification procedure where we attempt to classify all three stages without filtering out the NREM stage first. This one-phase procedure leads to significantly worse precision and recall values because it leaves more room for error between all three classes, whereas the two-phase process eliminates the epochs that are well distinguishable from the other ones first before it proceeds to the more difficult task of separating the two similar classes - REM and Wake.

\section{FUTURE WORK}

In the future, we plan to find good biophysical signals that can clearly separate the REM and Wake sleep stages to further improve the precision and reliability of the system. We also plan to develop a more fine-grained sleep stage identification framework where we can classify sleep epochs not only into Wake, REM and NREM but also into the different stages of NREM. Such a comprehensive sleep stage analysis system would enable a whole new range of applications. For hospital patients, for example, a fine-grained sleep staging framework can warn healthcare providers not to wake up sleeping patients, especially the elderly, during deep sleep stages to give them medication or for any other reasons. Waking up elderly people during the N3 stage of NREM can result in drowsiness and a greater likelihood for falls which are major causes of injury and mortality among elderly people [19]. This would require analyzing the pressure images in real time.

We would also like to study the effect of external stimuli, like sound, light, temperature and humidity on the sleep stage structure.

\section{CONCLUSION}

In this paper, we proposed an unobtrusive, completely wireless and contact-free sleep stage identification system.
We extracted a set of sleep-related biophysical features as well as geometric features from pressure images obtained from an e-textile bed sheet. These features were used as part of a twophase classification procedure that first separates NREM from REM and Wake and then separates the two latter classes. The superiority of the two-phase procedure over the onephase procedure was shown. The system achieved up to $70.3 \%$ precision and $71.1 \%$ recall on average.

The proposed unobtrusive system opens the way to a cheap contact-free sleep diagnosis solution, eliminating some of the drawbacks that the traditional PSG method presents. The combination of a flexible and noninvasive bed sheet system with simple signal processing and classification makes the described system a portable sleep screening solution that can be used in a clinical as well as a home environment. The affordability of the system can make sleep screening accessible to a bigger population which could lead to early diagnoses of sleep disorders and chronic diseases.

\section{ACKNOWLEDGMENT}

We would like to thank Dr. M. Woo, Professor at the UCLA School of Nursing, for providing the polysomnography equipment used in the experiments. We would also like to thank E. Valladares for providing many hours of useful training on using the equipment. We also thank X. Zhang for his help with editing and proofreading the paper.

\section{REFERENCES}

[1] (Access date: 2013, Apr.). Up24 by Jawbone [Online]. Available: http://jawbone.com/up

[2] (Access date: 2013, Apr.). British Snoring \& Sleep Apnoea Association [Online]. Available: http://www.britishsnoring.co.uk/why_do_i_snore/sleeping_position.php

[3] National Sleep Foundation, Arlington, VA, USA. (2012). Teeth Grinding [Online]. Available: http://www.sleepfoundation.org/article/sleeprelated-problems/bruxism-and-sleep

[4] (Access date: 2013, Mar.). Natural Patterns of Sleep [Online]. Available: http://healthysleep.med.harvard.edu/healthy/science//what/ sleep-patterns-rem-nrem

[5] (Access date: 2013, Mar.). Setting the Standard For Actigraphy [Online]. Available: http://www.healthcare.philips.com/main/homehealth//sleep/ actiwatch/default.wpd

[6] (Access date: 2013, Apr.). Sleep Tracker [Online]. Available: http://www.sleeptracker.com/

[7] Somnomedics, Coral Gables, FL, USA. (Access date: 2013, Mar.) [Online]. Available: http://www.somnomedics.eu/products/polysomnog raphy-18-33-channels.html

[8] Take Control of Your Sleep, King George, VA, USA. (Access date: 2013, Mar.) [Online]. Available: http://www.myzeo.com/sleep/

[9] J. Bak, N. Giakoumidis, G. Kim, H. Dong, and N. Mavridis, "An intelligent sensing system for sleep motion and stage analysis," in Proc. Int. Symp. Robot. Intell. Sensors, 2012, pp. 1-7.

[10] C. Bazil, "Specific sleep phenomena and sleep disorders confused with seizures," in Proc. Int. Symp. Robot. Intell. Sensors, 2005, pp. 1-10.

[11] H. Danker-Hopfe, P. Anderer, J. Zeitlhofer, M. Boeck, H. Dorn, and G. Gruber, "Interrater reliability (IRR) for sleep scoring according to the Rechtschaffen and Kalesandthe new AASM standard," J. Sleep Res., vol. 18, no. 1, pp. 74-84, 2009.

[12] L. de Souza, A. Benedito-Silva, M. Pires, D. Poyares, S. Tufik, and H. Calil, "Further validation of actigraphy for sleep studies," Sleep, vol. 26, no. 1, pp. 81-85, 2003.

[13] E. Estrada and H. Nazeran, "EEG and HRV signal features for automatic sleep staging and apnea detection," in Proc. 20th Int. Conf. Electron., Commun. Comput., Feb. 2010, pp. 142-147. 
[14] L. Fraiwan, K. Lweesy, N. Khazawneh, H. Wenz, and H. Dickhaus, "Automated sleep stage identification system based on time-frequency analysis of a single EEG channel and random forest classifier," Comput. Methods Program. Biomed., vol. 108, no. 1, pp. 10-19, 2011.

[15] J. Hedner, G. Pillar, S. Pittman, D. Zou, L. Grote, and D. White, "A novel adaptive wrist actigraphy algorithm for sleep-wake assessment in sleep apnea patients," Sleep, vol. 27, no. 4, pp. 1560-1565, 2004.

[16] E. Hoque, R. Dickerson, and J. Stankovic, Monitoring Sleep With WISP Tags. Charlottesville, VA, USA: Univ. Virginia, 2013.

[17] M.-C. Huang, W. Xu, J. Liu, L. Samy, A. Vajid, N. Alshurafa, et al., "Inconspicuous on-bed respiratory rate monitoring," in Proc. 6th Int. Conf. Pervas. Technol. Rel. Assist. Environ., 2013, pp. 1-3.

[18] C. Iber, S. Ancoli-Israel, A. Chesson, and S. Quan, The AASM Manual for the Scoring of Sleep and Associated Events: Rules Terminology and Technical Specifications. Grayslake, IL, USA: Amer. Acad. Sleep Med., 2007.

[19] P. Kannus, J. Parkkari, S. Niemi, and M. Palvanen, "Fall-induced deaths among elderly people," Amer. J. Public Health, vol. 95, no. 3 , pp. 422-424, 2005.

[20] J. Kantelhardt, T. Penzel, S. Rostig, H. Becker, S. Havlin, and A. Bunde, "Breathing during REM and non-REM sleep: Correlated versus uncorrelated behaviour," Physica, vol. 319, pp. 447-457, Jan. 2003.

[21] J. Kortelainen, M. Mendez, A. Bianchi, M. Matteucci, and S. Cerutti, "Sleep staging based on signals acquired through bed sensor," IEEE Trans. Inf. Technol. Biomed., vol. 14, no. 3, pp. 776-785, May 2010.

[22] J. J. Liu, W. Xu, M.-C. Huang, N. Alshurafa, M. Sarrafzadeh, N. Raut, et al., "A dense pressure sensitive bedsheet design for unobtrusive sleep posture monitoring," in Proc. IEEE Int. Conf. Pervas. Comput. Commun., Mar. 2013, pp. 207-215.

[23] M. Mendez, M. Migliorini, J. Kortelainen, D. Nistico, E. Arce-Santana, S. Cerutti, et al., "Evaluation of the sleep quality based on bed sensor signals: Time-variant analysis," in Proc. IEEE 32nd Annu. Int. Conf., Aug. 2010, pp. 3994-3997.

[24] J. Meyer, "Textile pressure sensor: Design, error modeling and evaluation," M.S. thesis, Dept. Comput Sci., ETH Zurich, Univ., Zurich, The Switzerland, 2008.

[25] Y. Peng, C. Lin, and M. Sun, "Multimodality sensors for sleep quality monitoring and logging," in Proc. 22nd Int. Conf. Data Eng. Workshops, 2006, pp. 1-108.

[26] J. Pilcher, D. Ginter, and B. Sadowsky, "Sleep quality versus sleep quantity: Relationships between sleep and measures of health, well-being and sleepiness in college students," J. Psychosomatic Res., vol. 42, no. 6, pp. 583-596, 1997.

[27] D. Purves, G. Augustine, and D. Fitzpatrick, Physiological Changes in Sleep States, 2nd ed. Washington, DC, USA: Neuroscience, 2001.

[28] S. Redmond and C. Heneghan, "Cardiorespiratory-based sleep staging in subjects with obstructive sleep apnea," IEEE Trans. Biomed. Eng., vol. 53, no. 3, pp. 485-496, Mar. 2006.

[29] A. Sadeh and C. Acebo, "The role of actigraphy in sleep medicine," Sleep Med. Rev., vol. 6, no. 2, pp. 113-124, 2002.

[30] M. Silber, S. Ancoli-Israel, M. Bonnet, S. Chokroverty, M. GriggDamberger, M. Hirshkowitz, et al., "The visual scoring of sleep in adults," J. Clin. Sleep Med., vol. 3, no. 2, pp. 121-131, 2007.

[31] M. Suetsugi, Y. Mizuki, K. Yamamoto, S. Uchida, and Y. Watanabe, "The effect of placebo administration on the first-night effect in healthy young volunteers," Progr. Neuro-Psychopharmacol. Biol. Psychiatry, vol. 31, no. 4, pp. 839-847, 2007.

[32] M. Thase, "Depression and sleep: Pathophysiology and treatment," Dialogues Clin. Neurosci., vol. 135, no. 2, pp. 217-226, 2006.

[33] W. Xu, Z. Li, M.-C. Huang, N. Amini, and M. Sarrafzadeh, "eCushion: An etextile device for sitting posture monitoring," in Proc. Int. Conf. BSN, 2011, pp. 194-199.

[34] W. Yao, C. Chu, and Z. Li, "The use of RFID in healthcare: Benefits and barriers," in Proc. IEEE Int. Conf. RFID-TA, Jan. 2010, pp. 128-134.

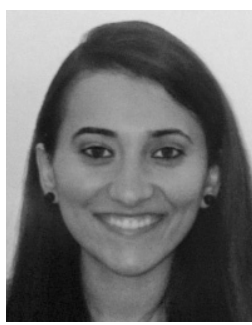

Lauren Samy is currently pursuing the $\mathrm{Ph} . \mathrm{D}$. degree at the Computer Science Department, University of California, Los Angeles, CA, USA. She received the B.S. degree in computer science from the University of California, Riverside, in 2011. Her research interests include embedded system design, data analytics, machine learning, and signal processing with an emphasis on applications in wireless health

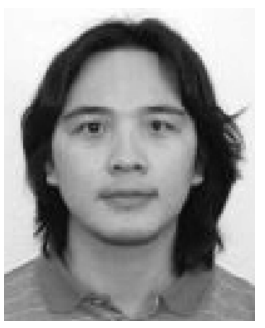

Ming-Chun Huang is currently pursuing the Ph.D. degree at the Computer Science Department, University of California, Los Angeles. He received the B.S degree in electrical engineering from National Tsing Hua University and the M.S. degree in electrical engineering from the University of Southern California. His research interests are in the area of medical sensor system design, computational modeling, and motivation-driven research, named data networking, and applications of smart infrastructure design.

He received the Best Medical and Performance Application Paper Award from the IEEE Conference on Implantable and Wearable Body Sensor Networks in 2013 and the Best Demonstration Award in ACM Wireless Health Conference in 2011. Teaching fellow position was granted for his long-term contribution of undergraduate education at UCLA in 2013. His research licensed to a start-up company of healthcare technology, Medisens. He has served as a Co-Founder and Senior Engineering Consultant with Medisens.

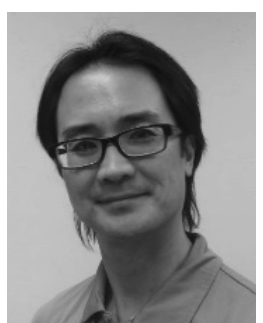

Jason J. Liu (S'10) received the B.Sc. degree in computer science and the B.E. degree in electrical engineering from the University of Sydney, Australia, and the M.S.E. degree in robotics from the University of Pennsylvania and is currently pursuing the Ph.D. degree in computer science at the University of California, Los Angeles. His research interests include computer vision, machine learning, medical informatics, wireless health, and humancomputer interaction.

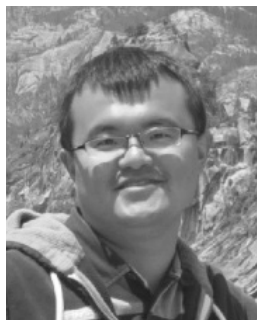

Wenyao Xu received the Ph.D. degree from the Electrical Engineering Department, University of California, Los Angeles, CA, USA, in 2013. Currently, he is an Assistant Professor with the Computer Science and Engineering Department, University at Buffalo, the State University of New York, New York, NY, USA. His current research interests include embedded system design, computational modeling, algorithm design, human computer interaction, integrated circuit design technologies, and their applications in medical and health applications. He received the Best Medical and Performance Application Paper Award from the IEEE Conference on Implantable and Wearable Body Sensor Networks in 2013 and the Best Demonstration Award from the ACM Wireless Health Conference in 2011.

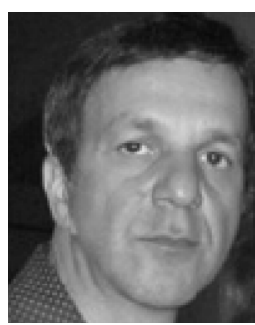

Majid Sarrafzadeh (M'87-SM'92-F'96) received his Ph.D. degree in 1987 from the University of Illinois at Urbana-Champaign in electrical and computer engineering. He joined Northwestern University as an Assistant Professor in 1987. In 2000, he joined the Computer Science Department at the University of California at Los Angeles (UCLA). $\mathrm{He}$ is a Co-Founder and Co-Director of the UCLA Wireless Health Institute (WHI). His recent research interests lie in the area of embedded computing with emphasis on healthcare. Dr. Sarrafzadeh is a Fellow of IEEE. Dr. Sarrafzadeh has published approximately 450 papers, co-authored 5 books, and is a named inventor on many U.S. patents. Dr. Sarrafzadeh has collaborated with many industries in the past 25 years. He co-founded two companies around 2000 - they were both acquired around 2004. $\mathrm{He}$ has recently co-founded two companies both in the area of technology in healthcare. 